Short Review

\title{
Reverse Mode Polymer Dispersed Liquid Crystal-based Smart Windows: A Progress Report
}

Yang Zhang ${ }^{1}$, Jiawen Chen ${ }^{1,2}$, Xiaowen Hu ${ }^{1,2}$, Wei Zhao ${ }^{1,2,{ }^{*}}$, Dirk J. Broer ${ }^{1,3}$, Guofu Zhou ${ }^{1,3,4}$

1. SCNU-TUE Joint Lab of Device Integrated Responsive Materials (DIRM), National Center for International Research on Green Optoelectronics, South China Normal University, No 378, West Waihuan Road, Guangzhou Higher Education Mega Center, 510006, Guangzhou China; E-Mails: ynnuzy@qq.com; j.chen@m.scnu.edu.cn; xwhu@m.scnu.edu.cn; weizhao@m.scnu.edu.cn; D.Broer@tue.nl; guofu.zhou@m.scnu.edu.cn

2. Guangdong Provincial Key Laboratory of Optical Information Materials and Technology \& Institute of Electronic Paper Displays, South China Academy of Advanced Optoelectronics, South China Normal University, Guangzhou 510006, P. R. China

3. Stimuli-responsive Functional Materials and Devices, Department of Chemical Engineering and Chemistry, Eindhoven University of Technology, Den Dolech 2, Eindhoven, 5600 MB, The Netherlands

4. Shenzhen Guohua Optoelectronics Tech. Co. Ltd., Shenzhen 518110, P. R. China

* Correspondence: Wei Zhao; E-Mail: weizhao@m.scnu.edu.cn

Academic Editors: Hassan-Ali Hakemi and Stanislaw J. Klosowicz

Special Issue: Polymer Dispersed Liquid Crystal Technology

Recent Progress in Materials

2021, volume 3, issue 4

doi:10.21926/rpm.2104044
Received: August 17, 2021

Accepted: October 14, 2021

Published: November 01, 2021

\section{Abstract}

The reverse mode polymer dispersed liquid crystal (PDLC) is an emerging smart window technology. Unlike traditional PDLCs, a reverse mode PDLC can be transparent and opaque in the absence and presence of an external electric field. This report provides a brief introduction to several reverse modes PDLC smart window technologies, focusing on polymer-stabilized liquid crystals (PSLCS). The systems based on electrohydrodynamic instability (EHDI) of liquid

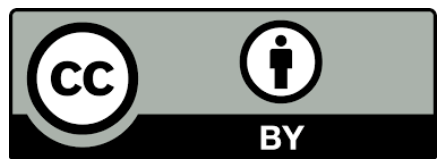

(C) 2021 by the author. This is an open access article distributed under the conditions of the Creative Commons by Attribution License, which permits unrestricted use, distribution, and reproduction in any medium or format, provided the original work is correctly cited. 
crystals have also been discussed. The working principles, mode of material design, and recent developments are presented for each technology. The current obstacles have also been pointed out. The prospects of smart windows have also been presented.

\section{Keywords}

Smart window; reverse mode polymer dispersed liquid crystal; polymer-stabilized liquid crystal; electrohydrodynamic instability

\section{Introduction}

A window is an indispensable part of modern residential buildings and vehicles. Windows directly influence the process of light and thermal management and human skin health [1,2]. Cutting-edge smart window technology involves the regulation of light of nearly all wavelengths by turning a knob or pressing a button [3]. According to the new Grand View Research report [4], in 2021, the global smart window market is valued at 5,683 million USD and is expected to nearly double to 9,983 million USD by 2025 . The compound annual growth rate is approximately $15.2 \%$ (Figure 1a). The ease of adjustability, the potential of individual control, and the maturity of the driving technology make the electro-smart window a mainstream product [5]. As pointed out by the Emergen Research [6], although an electrochromic smart window is still the first choice for the development of electrosmart windows, polymer dispersed liquid crystals (PDLCS) have gradually occupied a certain market share as they are characterized by fast response speed, high heat resistance, and wide temperature range (Figure $1 b$ ). The use of PDLCs has rapidly increased as they are used in buildings, projection screens, and luxury vehicles $[7,8]$.

a

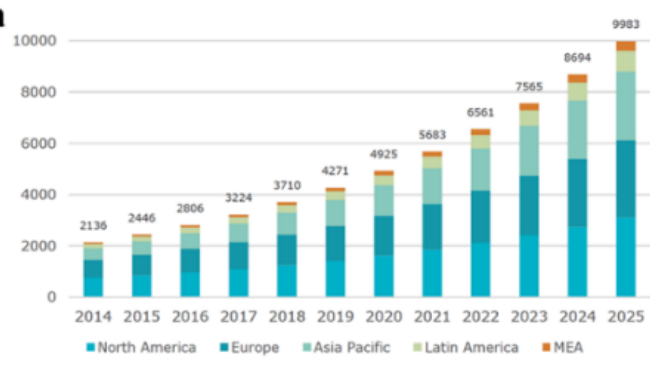

b

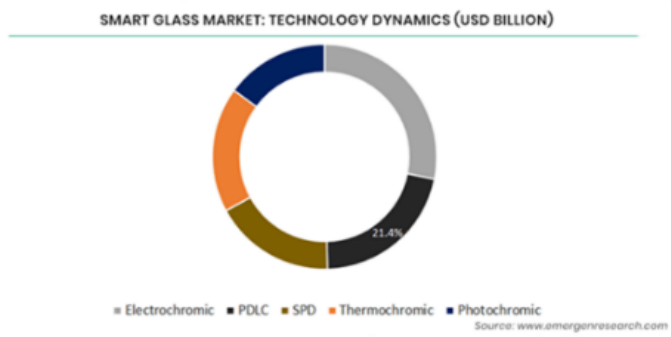

Figure 1 Smart window market. a) Growth in the market demand for smart windows in different regions worldwide from 2014 to 2025 [4]. b) Market share of various electrosmart windows [6]. 
PDLC was first discovered in the 1980s following a micro-emulsion method [9]. Later, J.W. Doane et al. prepared PDLC following a phase separation approach [10]. Since then, this method has been widely used in academic and industrial settings for the development of PDLC. In general, the amount of polymer content is greater than $40 \mathrm{wt}$ \% in PDLC. During the process of PDLC fabrication, the initial homogeneous mixture (consisting of curable monomers and LCs) is often subjected to heating conditions or irradiated with ultraviolet (UV) rays. Low-molar-mass LCs are typically insoluble in the polymer network, and thus they exist as isolated droplets embedded in a continuous polymer matrix. The PDLC material is sandwiched between two parallel transparent electrodes. A strong light scattering effect is produced as the refractive index of the randomly oriented LCs is different from that of the polymer matrix. The LC molecules in the droplets align along the direction of the electric field when an electric field is applied, causing the refractive index mismatch to disappear. Under these conditions, a transparent state is achieved [11-14]. At present, PDLC is a common commodity in the market and has been widely used for the fabrication of display units, smart windows, light shutters, and sensors $[15,16]$. However, a high driving voltage and continuous power are required to maintain the transparent state of PDLC. Thus, it is difficult to maintain a transparent state for a prolonged period of time. Thus, PDLC cannot be effectively used to fabricate windows used in residential buildings and vehicles [17]. The view angle of PDLC at the transparent state is generally poor. This can be attributed to the fact that the refractive indices of the LCs and polymers do not match perfectly. Therefore, a reverse mode PDLC that remains transparent in the OFF state and can be turned into an opaque (ON) state is highly desirable.

Researchers have tried to fabricate reverse mode PDLC [18] using dual-frequency LCs [19], realizing homogenous/homeotropic alignment of the substrate surface [20] and modifying the surface energy of polymer droplets [21]. In this progress report, we have focused on the work conducted by our group to develop reverse mode PDLC. Specifically, we have focused on polymerstabilized liquid crystals (PSLCS) and electrohydrodynamic instability (EHDI) techniques. We will briefly discuss the working principles of these techniques and present a part of the current and ongoing research being conducted in our group. Finally, the opportunities and challenges in the field of developing reverse mode PDLC technique has been pointed out. The prospects have also been mentioned.

\section{Reverse Mode PDLCs}

Reverse mode PDLC-based smart windows that are driven by electric fields typically consist of LC materials sandwiched between transparent conductive electrodes. These can reversibly switch their optical properties between a clear OFF state and an opaque ON state. The transparent OFF state of the reverse mode PDLC smart windows is comparable to normal glasses characterized by low haze. When powered on, they can generate an opaque state that is comparable to PDLC, and good privacy protection can be achieved. Depending on the mechanism, the technologies can be divided into three main groups.

\subsection{Polymer Stabilized Liquid Crystals (PSLCS)}

PSLC contains a low concentration of LC polymer network ( $<10 \mathrm{wt} . \%)$ embedded in a continuous LC matrix [22]. The pioneering work was done by Broer in 1989 [23] and Hikmet in 1990 [24]. Both the researchers worked in the research group headed by Philips. During the process of 
polymerization, the polymer network in the PSLC system forms a template of the LCs (in the order in which it was created) [25]. In the absence of an electric field, the negative dielectric LC (i.e., an LC of negative dielectric anisotropy) and the polymer network are orientated perpendicularly to the substrate. This can be attributed to the homeotropic alignment of the layers on both sides. In such cases, the incident light passes through the PSLC, and the device remains in a transparent state. When an electric field is applied, the negative dielectric LCs tend to orient in the direction parallel to the substrate. Under these conditions, the polymer network hinders the rotation of the system, and a multi-domain structure is thus formed. The incident light gets scattered and the device becomes opaque (Figure 2). As the polymer content is low, LC molecules can easily break the initial constraints of the polymer network and start rotating under conditions of low electric field strength, resulting in the generation of a low operating voltage. Positive dielectric LCs can also be used to fabricate PSLC-based smart windows, which operate following a similar reverse mode. The homogeneous alignment boundary conditions are needed [26]. When cholesteric LCs are used instead of nematic LCS, the PSLC device can also provide the desired reverse mode function [27]. Due to the limited scope of this progress report, these two types of PSLCs will not be extensively discussed. Only one example based on the cholesteric LCs has been provided in Section 2.3.

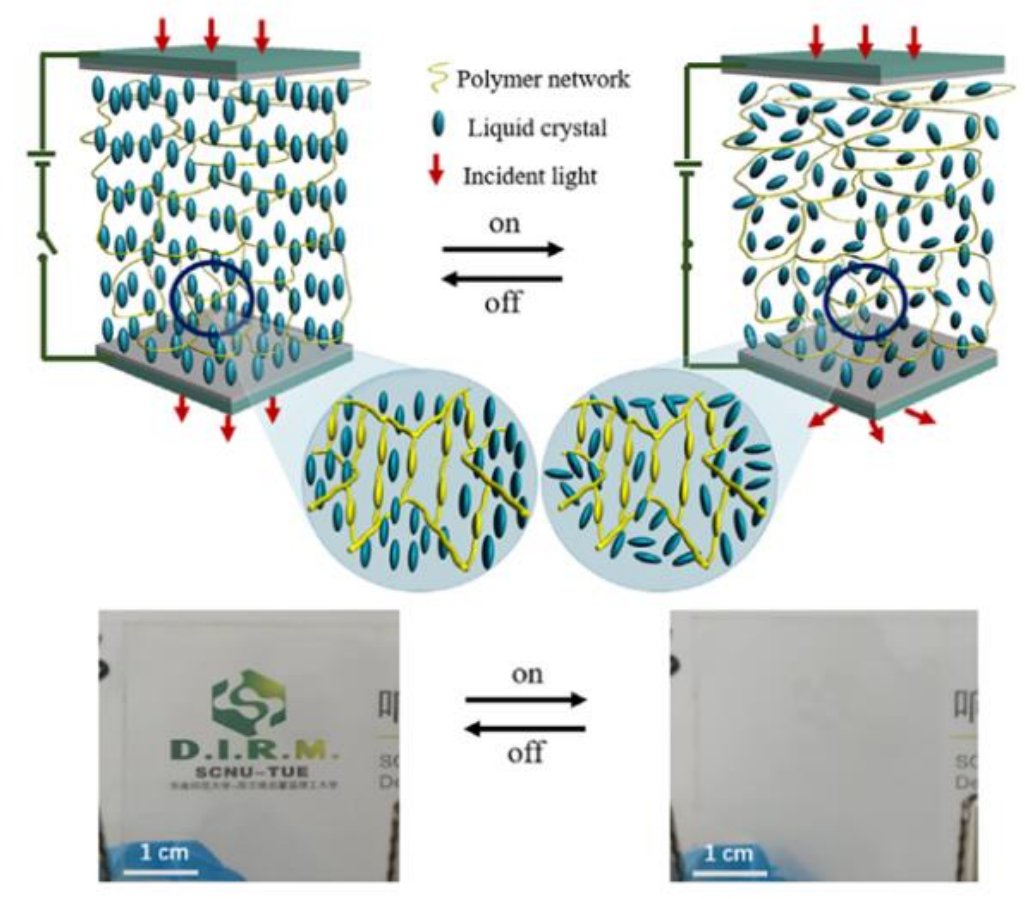

Figure 2 Schematic representation of the working principle and images of PSLCs in their OFF/ON states [28].

Although PSLC exhibits interesting electro-optical properties, the properties need to be improved further. The topology of the polymer network in a PSLC plays a crucial role in determining the electro-optical properties. Zhou et al. mixed a mono-acrylate monomer with the normally used diacrylate monomer to regulate the polymer network structure [29]. As the ratio of the mono-acrylate monomer increases, the polymer network gradually becomes softer and less dense following the process of photo-polymerization (Figure 3a). The PSLCs can function properly under these 
conditions and result in scattering. The transmittance drops in the presence of the field. The operating voltage decreases from 17.4 to $7.5 \mathrm{~V}$ when the concentration of the mono-acrylate increases from 0 to $2.4 \%$ (Figure $3 \mathrm{~b}$ ). Under these conditions, the polymer network turns soft, and its ability to bind LCs is weakened. Even though the introduction of mono-acrylate can reduce the operating voltage, the introduction softens the polymer network, making it more prone to fatigue. This is not ideal for prolonged applications.

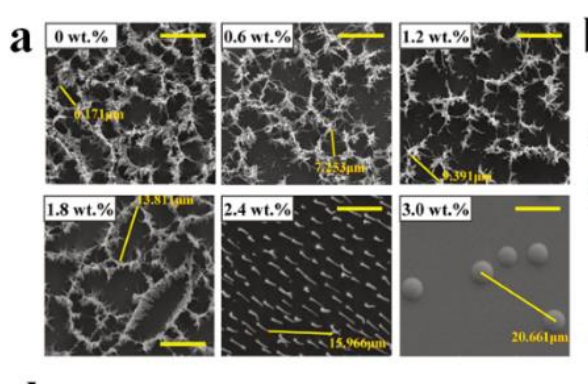

d

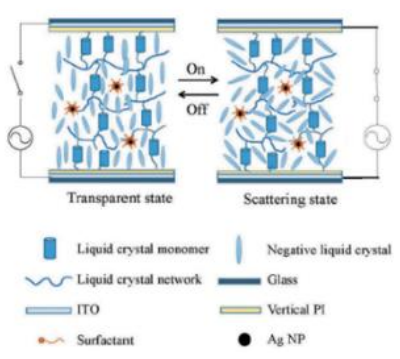

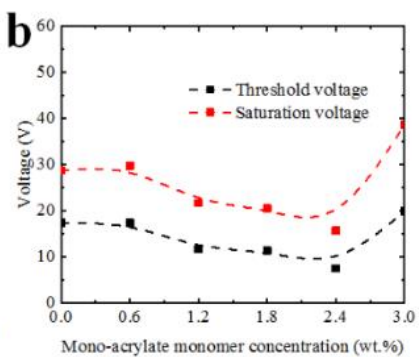

f
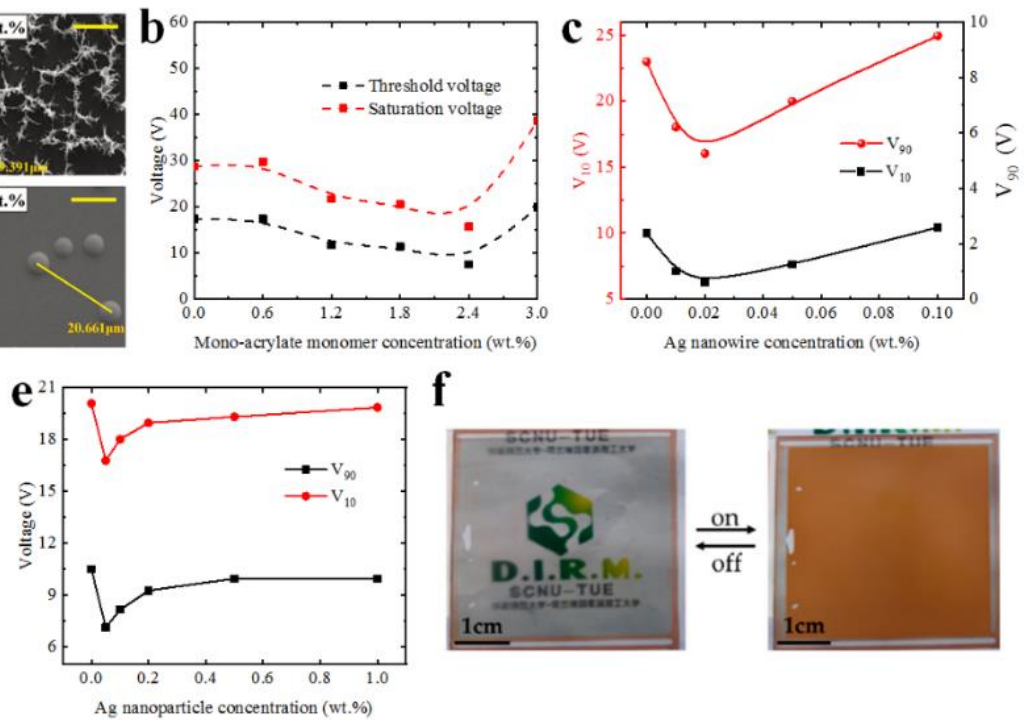

Figure 3 a) Top-view of the SEM images of the morphology of the polymer network under conditions of varying mono-acrylate monomer ratios [29]. b) The threshold and saturation voltage for PSLC under conditions of varying mono-acrylate monomer ratios [29]. c) Variations in the voltage for $10 \%$ transmission $\left(V_{10}\right)$ and $90 \%$ transmission $\left(V_{90}\right)$ under conditions of varying $\mathrm{Ag}$ nanowire concentrations [30]. d) Schematic representation of the working principle of PSLC doped with $\mathrm{Ag}$ nanoparticles in the OFF/ON states [31]. e) Variations in the voltages for $V_{10}$ and $V_{90}$ under conditions of varying Ag nanoparticle concentrations [31]. f) The image of a colored PSLC in the OFF/ON states [32].

The electro-optical properties of PSLC can also be improved by doping. Yan et al. doped Ag nanowires into PSLC [30]. When the concentration of the Ag nanowire increases from 0 to $0.02 \mathrm{wt} . \%$, the electro-optical properties improve (especially the threshold voltage decreases from 2.4 to $0.6 \mathrm{~V}$ ) (Figure 3c). Ag nanowires are found to significantly enhance the ionic purity of LCs, resulting in the reduction in the conductivity of the active PSLC layer. Under these conditions, an increase in the strength of the applied force (applied on the LC molecules) is observed when the field is on. An overlap between the Ag nanowires and short-circuit occurs when the nanowire concentration is increased further. The conductivity also increases under these conditions. The Ag nanoparticles coated with a single layer of the surfactant sodium dodecyl sulfate (SDS) to form inverse micelles can also be doped into PSLCs to tune the electro-optical performance of the system [31]. As the flexible tail of the LC molecule and the SDS molecule can be directionally coupled, the randomly dispersed reverse micelles cause local distortion in the LC alignment (Figure 3d). Therefore, the LC molecules can easily deviate from the anchoring direction of the homeotropic alignment and rotate 
when the power is on. It is observed that doping with a small concentration of $\mathrm{Ag}$ nanoparticles (only 0.05 wt.\%) can effectively lower the threshold voltage by $39 \%$ for the PSLC (from $11.5 \mathrm{~V}$ to 7.1 V) (Figure 3e). the appropriate doping concentration should be carefully controlled.

The PSLCs presently in use are primarily colorless and are characterized by low contrast. Sun et al. reported that the process of direct doping with non-dichroic dyes produces colored OFF/ON states. This is not beneficial for increasing the contrast of PSLC [32]. When doped with a dichroic dye, PSLC shows a less intense color in the OFF state and a highly intense color in the ON state, resulting in the generation of high contrast. The high contrast can be attributed to the orientation-dependent absorption band of the dichroic dye. Furthermore, the mixing of the non-dichroic and dichroic dyes characterized by complementary absorption bands can eliminate the color of PSLC in the OFF state following the process of subtractive color mixing. This results in realizing better contrast (Figure 3f). It is predicted that colored PSLCs can be effectively used in various fields. They can be used for the fabrication of smart windows. Nevertheless, the process of subtractive color mixing involves precise tuning of the absorption band and the concentration of the dichroic and non-dichroic dyes. It is not easy to fabricate a black PSLC smart window.

In recent years, flexible optoelectronic devices have received significant attention as they are lightweight, cost-effective, thin, and easy to use [33]. The market trends demand that flexible PSLC films should be developed. Nevertheless, various problems should be addressed to fabricate a flexible PSLC film: (i) a homeotropic alignment layer should be developed on the flexible substrate, i.e., PET; (ii) the extent of adhesion between the polymer network and the conductive substrates (include the alignment layer) should be increased; (iii) the mechanical strength of the polymer network should be improved to prevent breakage during the process of bending while maintaining the electro-optical properties of PSLC. The polyimide homeotropic alignment layer of a PSLC is usually obtained by heating the polyamide acid (PAA) at a temperature $>200{ }^{\circ} \mathrm{C}$. This limits the fabrication of PSLC on the flexible films, as they usually melt or degrade at such high temperatures. Zhang et al. reported that low-temperature (incomplete imidization) treatment of PAA neither negatively affects the homeotropic alignment of the LCs, nor impacts the formation of a polymer network on PSLC [34] (Figure 4a). The results presented herein lay a solid foundation for the development of flexible PSLC films. Zhao's group uses bi-functional silanes to introduce chemical bonds between the polymer network and the alignment layer. This could address the problem presented in (ii) (Figures $4 \mathrm{~b}$ and $4 \mathrm{c}$ ). The preliminary results indicate improved adhesion strength at the interface [35]. We believe that once successfully developed, flexible PSLC films shall accelerate the advancement of the field related to smart windows. 

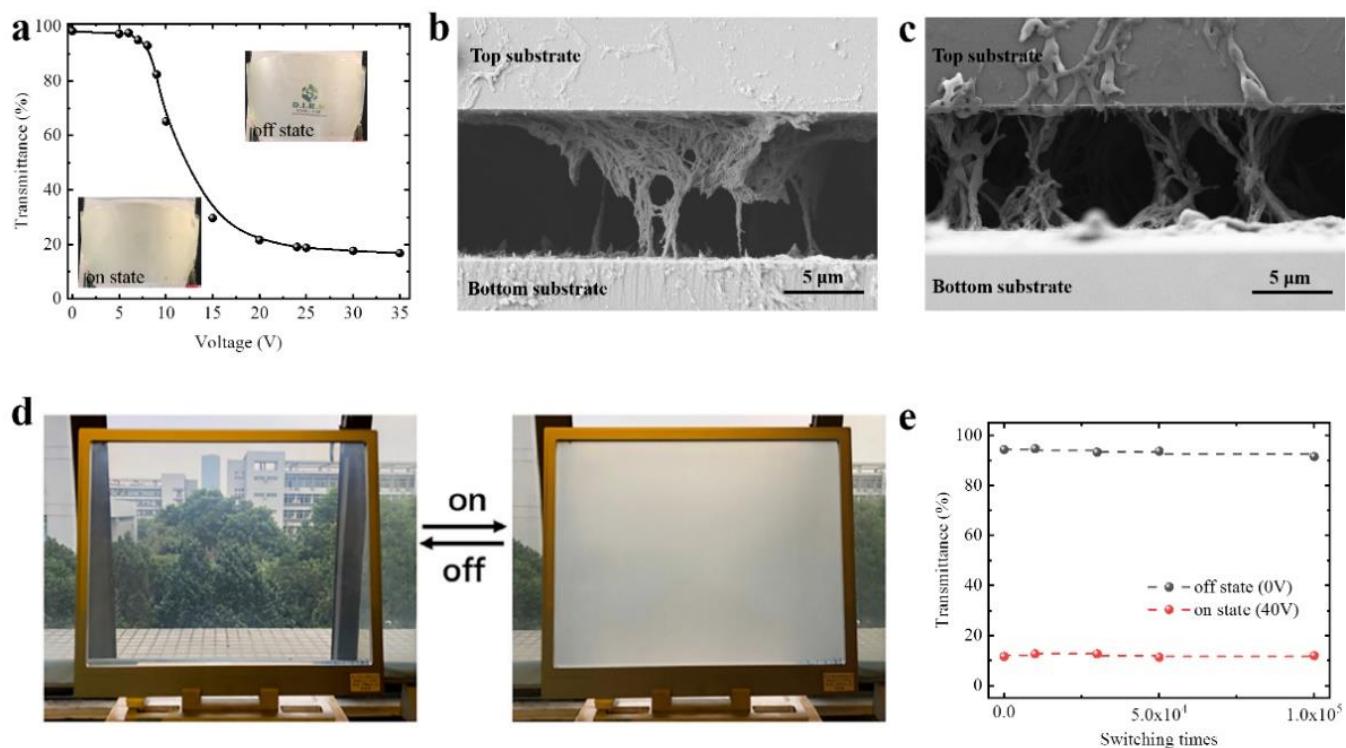

Figure 4 a) Electro-optical performance and images of PSLC fabricated on the flexible substrate in the OFF/ON states [34]. Cross-sectional SEM images of the polymer networks (for PSLC) with alignment layers fabricated using b) polyimide and c) bifunctional silanes [35]. d) Prototype of a G2.5 size PSLC smart window in the OFF/ON state [28]. e) Transmittance recorded for a PSLC in the OFF and ON states as a function of switching times [28].

The industrial-scale fabrication of PSLCs has been achieved by our group. A large $\left(40 \times 50 \mathrm{~cm}^{2}\right)$ PSLC-based smart window has been developed in the generation 2.5 (G2.5) production line as reported by $\mathrm{Hu}$ et al. (Figure 4d) [28]. The fabricated PSLC exhibits excellent electro-optical performance and switches at least 100,000 times without any change. It can be potentially commercialized (Figure 4e).

\subsection{Electrohydrodynamic Instability of Liquid Crystals (EHDI)}

The major disadvantage of using a PSLC is the fatigue of the polymer network. Researchers have hence used the polymer-free smart window technology based on the electrohydrodynamic instability (EHDI) of LCS for device fabrication. EHDI was first discovered in the 1960s (earlier than PDLC and PSLC) for the fabrication of display units [36]. The structure of an EHDI-based device is quite simple. It contains ionic impurities added to the aligned LCs. When the applied electric field strength is high enough (only a few tens of volts), the dynamic scattering mode is generated. Under these conditions, turbulence is also induced by ion movement between parallel electrodes, resulting in strong light scattering (Figure 5a). Unlike the PSLC smart windows, the EHDI system does not contain polymers. This eliminates the polymerization step and improves the lifetime of the device. It has been reported that such devices can be switched thousands of times without causing a degradation in the electro-optical properties. 

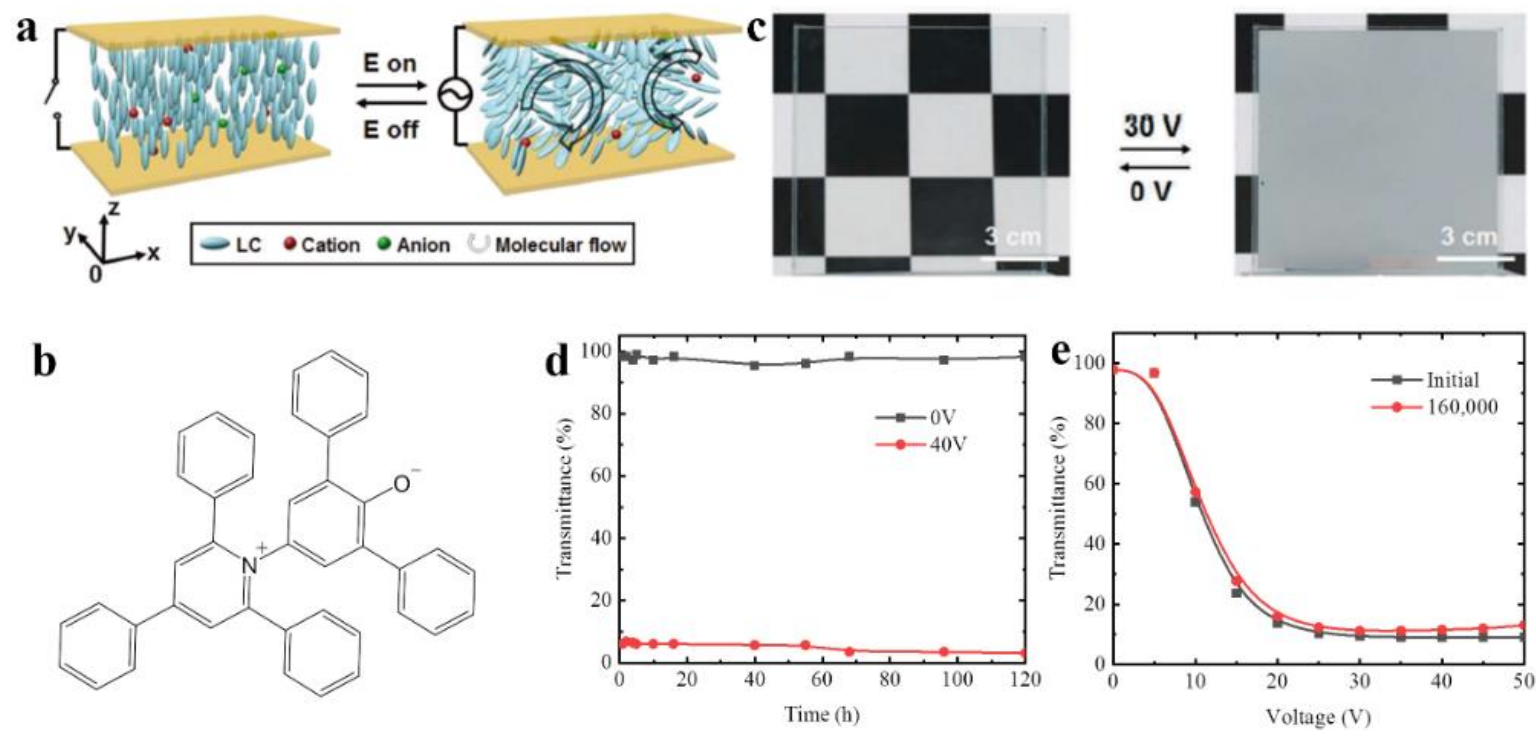

Figure 5 a) Schematic representation of the operating principle of the EHDI smart window in the OFF/ON states [37]. b) Chemical structure of the zwitterionic Reichardt's dye [37]. c) Image of the EHDI based on the zwitterion [37]. EHDI smart window fabricated using negative dielectric LCs doped with $0.1 \mathrm{wt} . \%$ of the zwitterion in the homeotropic cell. d) Transmittance under the on/off states under conditions of continuous power and e) voltage-transmittance curves recorded before and after switching (160,000 times) [38].

However, ion aggregation and degradation restrict the development of EHDI technology. The moderate solubility of electrolytes in LCs and accumulation of ions onto electrodes results in the reduction in the lifetime of the EHDI devices. To solve these problems, Zhan et al. proposed to replace the traditional electrolytes with zwitterions [37]. Zwitterions are molecules with multiple ionic groups. At least one of the groups is positively charged, and one of the groups is negatively charged. The whole molecule remains charge-neutral (Figure 5b). The replacement of the ionic dopant in the EHDI smart window can potentially result in the reduction in the extent of accumulation of the ions on the electrodes (Figure 5c). Based on this, Zhang et al. explored the EHDI phenomena by doping the zwitterionic Reichardt's dye into the dielectrically positive/negative host LCs. The process was performed under conditions of the boundary conditions (homogenous/homeotropic alignment) [38]. The results revealed the generation of numerous novel EHDI phenomena when zwitterions were used as the ionic impurities. The EHDI smart window based on the zwitterions maintains excellent performance even under conditions of continuous power application (Figure $5 \mathrm{~d}$ ) and tens of thousands of ON/OFF switching modes (Figure 5e). In the presence of dichroic dyes, EHDI smart windows can also be used to achieve a colored scattering state characterized by enhanced contrast [39]. The simple mechanism of action of the EHDI smart windows helps achieve design and control flexibility (such as remote control via light) [40]. In addition to nematic LCs, ion-doped smectic LCs have been recently used to prepare "write-erase" smart windows $[41,42]$.

Overall, EHDI is better than PSLC in terms of the nature of raw materials used, ease of preparation, and ease of scale-up. However, as EHDI is based on the movement of ionic species and non-negligible current is produced during the power "on" state, a drop in voltage (for the conductive electrodes) 
hinders the fabrication of a large-sized smart window. Research efforts are still needed to further this technology.

\subsection{Other Technologies}

In addition to nematic LCs, other types of LCs can also be used for the fabrication of smart windows that switch between a clear OFF state and an opaque ON state. The cholesteric LCs (CLCs), known to produce a haze-free state when homogeneously aligned. They turn into a scattering focalconic state under conditions of moderate voltage, as demonstrated in the pioneering work by Yang et al. [43]. The CLC system is characterized by unique properties. The intrinsic color can be attributed to the periodic helical structure and the potential bi-stability of both the planar and focal-conic states. However, some immediate problems hinder its application. The instability of the focal-conic texture and the lack of a convenient means to switch between the focal-conic and the planar textures hinder the application. The strong angular dependence of the reflected light intensity also poses a problem. To solve this problem, a low concentration of the curable monomer was introduced and polymerized by Yang et al. [44]. Taking advantage of the CLC systems, Mo et al. developed a smart window with four stable optical states [45]. The designed smart window can switch between the colored transparent state (reflective state), the colored opaque state (reflective and scattering co-existing state), the colorless opaque state (scattering-only state), and the colorless transparent state (transparent non-reflective state) (Figure 6a) when a polymer-stabilized CLC and a small molecular CLC layer are formed separately. The maintenance of three of these four states requires no power. Thus, good energy efficiency can be achieved. Wang et al. also explored chiral LC oligomers as chiral dopants to realize the improved electro-optical performance of the CLC-based smart windows [46]. The chiral oligomers form during photo-polymerization, which could be monitored in-situ by analyzing the shift in the CLC reflection peak. The results provide a method to study the relationship between the structure of the chiral dopants and device performance (Figures $6 \mathrm{~b}$ and $6 \mathrm{c})$. Other compounds, including chiral LC dimers and trimers, are also explored in terms of their potential to extend the lifetime of the pseudo-stable focal-conic state. 


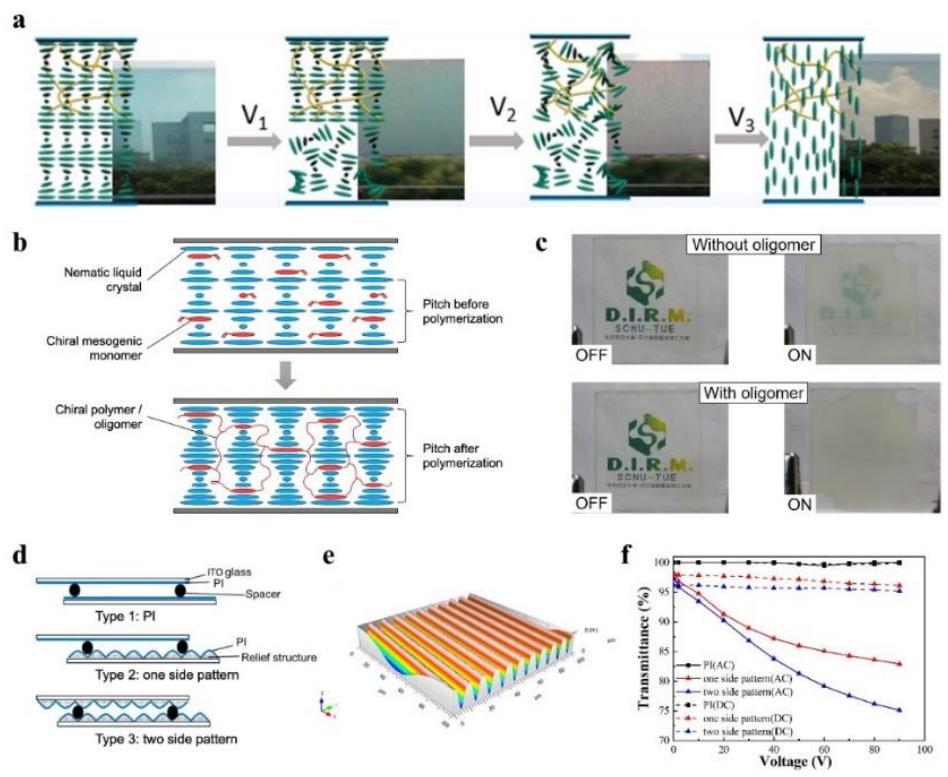

Figure 6 a) Schematic representation of the working principle and images of the multistable CLC windows with four optical states based on the bilayer structure consisting of a polymer-stabilized CLC layer and a non-polymer stabilized CLC layer [45]. b) Schematic representation showing the increase in the cholesteric pitch upon polymerization of the chiral LC monomers [46]. c) Images of the CLC smart windows at the OFF/ON states in the absence and presence of chiral oligomers [46]. d) Three types of LC cells fabricated with different alignment layers [47]. e) Topography of the photo embossed relief structure [47]. f) Electro-optical properties of smart windows fabricated under conditions of alignment designs as shown in (d). Comparison of the results obtained for devices driven by AC or DC [47].

To understand the polymer-free approach, another strategy based on LC cells with corrugated inner surfaces has been studied. Photo-embossing was adopted as the method to prepare such corrugated surface structures. This process has been reported to be simple and efficient [48]. When a line pattern is introduced at the interface between the LCs and the alignment layer, the transmittance of the device drops continuously when the voltage is increased. The maximum drop in transmittance (to approximately $75 \%$ ) was observed when both sides of the LC cell exhibited an undulating line pattern. Thus, it was a viable design for the fabrication of a light-regulating smart window (Figures 6d-f) [47].

\section{Summary}

This progress report describes the recent advances made in the field of reverse mode PDLC smart window technologies. We have emphasized the work conducted in our group. We focused on two main strategies (PSLC and EHDI) and briefly described some of the other methods used for fabrication. The working mechanisms for each category were introduced, and the techniques and materials that improve the performances of the smart windows were presented. A number of issues must be addressed before the reverse mode PDLC smart window technologies can be used for fabricating windows for buildings and automobiles. (i) The durability of smart windows, especially 
when in contact with outdoor conditions, should be improved by paying attention to material selection and structural design. Transparent anti-UV coating technology can be used to coat the surface of the PSLC substrates to prevent the aging of the polymer network in contact with the outdoor environment. (ii) For economic reasons, new production techniques should be developed. The introduction of a slicing technology that can divide large display screens into smart windows may alleviate the limitation of production line size and address the problem that hinders the development of large EHDI smart windows. A flexible smart window based on roll-to-roll technology is urgently needed for practical use. (iii) The introduction of additives can help in advancing the applications of smart windows. The development of reverse mode PDLC smart windows can fully use the functional LC materials by broadening the stimulation methods. The windows can be used to protect privacy, and the materials can also be used to fabricate sensors. A good example is the patterned EHDI smart window that, in addition to its electric control, can respond to light of various wavelengths [40]. (iv) The amount of energy consumed by the smart windows should be controlled to address the problem of global energy shortage and carbon neutrality. Smart windows that can be fabricated using active energy generators such as solar cells or triboelectric nanogenerator (TENG) to realize self-powered smart windows are highly desirable. In short, the emergence of smart windows has already broken the definition of traditional windows. The reverse mode PDLC smart windows, especially the ones that integrate various functions, will be in high demand in the near future.

\section{Acknowledgments}

This work was supported financially by National Key R\&D Program of China (No. 2020YFE0100200), Special Projects in Key Areas of Guangdong Provincial Department of Education (No. 2020ZDZX2064), Science and Technology Program of Guangzhou (No. 2019050001), Guangdong Provincial Key Laboratory of Optical Information Materials and Technology (No. 2017B030301007), MOE International Laboratory for Optical Information Technologies, the 111 Project and Yunnan expert workstation (No. 202005AF150028).

\section{Author Contributions}

Y Zhang: Investigation, software, writing- review \& editing. J Chen: Data curation, methodology, supervision. X Hu: Software, resources. W Zhao: Conceptualization, supervision, writing- review \& editing. D J. Broer: Conceptualization, writing- review \& editing. G Zhou: Funding acquisition.

\section{Competing Interests}

The authors have declared that no competing interests exist.

\section{References}

1. Rezaei SD, Shannigrahi S, Ramakrishna S. A review of conventional, advanced, and smart glazing technologies and materials for improving indoor environment. Sol Energy Mater Sol Cells. 2017; 159: 26-51.

2. Edlich RF, Winters KL, Cox MJ, Becker DG, Edlic EC. Use of UV-protective windows and window films to aid in the prevention of skin cancer. J Long Term Eff Med Implants. 2004; 14: 415-430. 
3. Mohamed AS. Smart materials innovative technologies in architecture; towards innovative design paradigm. Energy Procedia. 2017; 115: 139-154.

4. Liettyä J. Smart glass makes homes sustainable - not just impressive. Finland: Glastory; 2021. Available from: https://www.glastory.net/smart-glass-makes-homes-sustainable/.

5. Casini M. Active dynamic windows for buildings: A review. Renew Energy. 2018; 119: 923-934.

6. Smart glass market by application (architectural, transportation, consumer electronics, power generation, others), by technology (electrochromic, PDLC, SPD, thermochromic, photochromic) and by regions forecasts to 2027. British Columbia: Emergen Research; 2020. Available from: https://www.emergenresearch.com/industry-report/smart-glass-market.

7. Hakemi HA, Pinshow O, Gal-Fuss D. Evaluation of polymer dispersed liquid crystal (PDLC) for passive rear projection screen application. Recent Prog Mater. 2019; 1: 1-15.

8. Guo S, Liang X, Zhang H, Shen W, Li C, Wang X, et al. An electrically light-transmittancecontrollable film with a low-driving voltage from a coexistent system of polymer-dispersed and polymer-stabilised cholesteric liquid crystals. Liq Cryst. 2018; 45: 1854-1860.

9. Fergason JL. Encapsulated liquid crystal and method. US Patent 4435047 [Internet]. United States Patent; $1984 . \quad$ Available from: https://patentimages.storage.googleapis.com/71/2a/e3/14a3e9f1a6a992/US4435047.pdf.

10. Doane JW, Chidichimo G, Vaz NA. Light modulating material comprising a liquid crystal dispersion in a plastic matrix. US Patent 4688900 [Internet]. United States Patent; 1987. Available from: https://patentimages.storage.googleapis.com/11/5e/4c/0f243220bb99c3/US4688900.pdf.

11. Bouteiller L, Barny PL. Polymer-dispersed liquid crystals: Preparation, operation and application. Liq Cryst. 1996; 21: 157-174.

12. Bronnikov S, Kostromin S, Zuev V. Polymer-dispersed liquid crystals: Progress in preparation, investigation, and application. J Macromol Sci Phys. 2013; 52: 1718-1735.

13. Higgins DA. Probing the mesoscopic chemical and physical properties of polymer-dispersed liquid crystals. Adv Mater. 2000; 12: 251-264.

14. Hakemi $\mathrm{H}$. The effect of curing temperature and time on morphology and Electro-Optical properties of flexible thermoset polymer dispersed liquid crystal (PDLC) films. Mol Cryst Liq Cryst. 2020; 703: 1-12.

15. Li C, Chen M, Zhang L, Shen W, Liang X, Wang X, et al. An electrically light-transmittanceswitchable film with a low driving voltage based on liquid crystal/polymer composites. Liq Cryst. 2020; 47: 106-113.

16. Edwards R, Ward J, Zhou L, Trushkevych O. The interaction of polymer dispersed liquid crystal sensors with ultrasound. Appl Phys Lett. 2020; 116: 044104.

17. MacChione M, Cupelli D, Filpo GD, Nicoletta FP, Chidichimo G. Morphology and electro-optical properties of reverse mode polymer dispersed liquid crystals. Liq Cryst. 2000; 27: 1337-1341.

18. Ahmad F, Jamil M, Jeon YJ. Current trends in studies on reverse-mode polymer dispersed liquidcrystal films-a review. Electron Mater Lett. 2014; 10: 679-692.

19. Gotoh T, Murai H. Preparation and characteristics of new reverse mode film of polymer dispersed liquid crystal type. Appl Phys Lett. 1992; 60: 392-394.

20. Chen TJ, Chen YF, Sun $\mathrm{CH}$, Wu JJ. Electro-optical properties of reverse-mode films of planar aligned polymer-dispersed liquid crystal. J Polym Res. 2006; 13: 85-89.

21. Ma YD, Wu BG, Xu G. Reverse-mode microdroplet liquid crystal display. Liq Cryst Disp Appl. 1990; 
1257: 46-57.

22. Zhang $Y$, Yang W, Zhao W, Li M, Zhou G. Research advance in polymer stabilized liquid crystals. J Funct Polym. 2021; 34: 49-65.

23. Broer DJ, Boven J, Mol GN, Challa G. In-situ photopolymerization of oriented liquid-crystalline acrylates, $3^{\dagger}$. Oriented polymer networks from a mesogenic diacrylate. Macromol Chem Phys. 1989; 190: 2255-2268.

24. Hikmet R. Electrically induced light scattering from anisotropic gels. J Appl Phys. 1990; 68: 44064412.

25. Dierking I. Polymer-modified liquid crystals. London, UK: Royal Society of Chemistry; 2019.

26. Fan YH, Ren $\mathrm{H}, \mathrm{Wu}$ ST. Switchable Fresnel lens using polymer-stabilized liquid crystals. Opt Express. 2003; 11: 3080-3086.

27. Ren H, Wu ST. Reflective reversed-mode polymer stabilized cholesteric texture light switches. J Appl Phys. 2002; 92: 797-800.

28. Hu X, Zhang X, Yang W, Jiang XF, Jiang X, de Haan LT, et al. Stable and scalable smart window based on polymer stabilized liquid crystals. J Appl Polym Sci. 2020; 137: 48917.

29. Zhou Y, You Y, Liao X, Liu W, Zhou L, Zhang B, et al. Effect of polymer network topology on the electro-optical performance of polymer stabilized liquid crystal (PSLC) devices. Macromol Chem Phys. 2020; 221: 2000185.

30. Yan X, Liu W, Zhou Y, Yuan D, Hu X, Zhao W, et al. Improvement of electro-optical properties of PSLC devices by silver nanowire doping. Appl Sci. 2019; 9: 145.

31. Yan X, Zhou Y, Liu W, Liu S, Hu X, Zhao W, et al. Effects of silver nanoparticle doping on the electro-optical properties of polymer stabilized liquid crystal devices. Liq Cryst. 2020; 47: 11311138.

32. Sun H, Xie Z, Ju C, Hu X, Yuan D, Zhao W, et al. Dye-doped electrically smart windows based on polymer-stabilized liquid crystal. Polymers. 2019; 11: 694.

33. Sivaranjini B, Mangaiyarkarasi R, Ganesh V, Umadevi S. Vertical alignment of liquid crystals over a functionalized flexible substrate. Sci Rep. 2018; 8: 1-13.

34. Zhang $Y$, Wang $C$, Zhao W, Li M, Wang $X$, Yang $X$, et al. Polymer stabilized liquid crystal smart window with flexible substrates based on low-temperature treatment of polyamide acid technology. Polymers. 2019; 11: 1869.

35. Zhang $Y$, Yang $W, G u$ M, Wei Q, Lv P, Li M, et al. Versatile homeotropic liquid crystal alignment with tunable functionality prepared by one-step method. J Colloid Interface Sci. 2021.

36. Williams R. Domains in liquid crystals. J Chem Phys. 1963; 39: 384-388.

37. Zhan $Y$, Lu H, Jin M, Zhou G. Electrohydrodynamic instabilities for smart window applications. Liq Cryst. 2020; 47: 977-983.

38. Zhang $Y$, Yang $X$, Zhan $Y$, Zhang $Y$, He J, Lv P, et al. Electroconvection in zwitterion-doped nematic liquid crystals and application as smart windows. Adv Opt Mater. 2021; 9: 2001465.

39. Zhou B, Che C, Li Y, Wang C, Wu Y, Chang W, et al. Dielectric reorientation and electrohydrodynamic instabilities in dichroic-dye-doped dual-cell liquid crystal smart window. Liq Cryst. 2021. doi: 10.1080/02678292.2021.1945692.

40. Zhan Y, Schenning AP, Broer DJ, Zhou G, Liu D. Light-driven electrohydrodynamic instabilities in liquid crystals. Adv Funct Mater. 2018; 28: 1707436.

41. Mrukiewicz M, Perkowski P, Urbańska M, Węgłowska D, Piecek W. Electrical conductivity of iondoped fluoro substituted liquid crystal compounds for application in the dynamic light scattering 
effect. J Mol Liq. 2020; 317: 113810.

42. Guo $S$, Liang $X$, Wang $M$, Zhang $C$, Zhang L, Yang H. Periodic electro-optical characteristics of iondoped Smectic A phase liquid crystals driven by a low-frequency electric field. Liq Cryst. 2019; 46: 905-912.

43. Yang DK, Chien LC, Doane JW. Cholesteric liquid crystal/polymer dispersion for haze-free light shutters. Appl Phys Lett. 1992; 60: 3102-3104.

44. Yang DK, West JL, Chien LC, Doane JW. Control of reflectivity and bistability in displays using cholesteric liquid crystals. J Appl Phys. 1998; 76: 1331-1333.

45. Mo L, Sun $H$, Liang A, Jiang $X$, Shui L, Zhou G. Multi-stable cholesteric liquid crystal windows with four optical states. Liq Cryst. 2021. doi: 10.1080/02678292.2021.1962422.

46. Wang $X$, He J, Wei $Q$, Zhang $Y$, Li Y, Zhang Z, et al. Influence of molecular weight on helical twisting power of oligomer chiral dopants. J Mol Liq. 2021; 339: 116816.

47. Sánchez C, de Gans BJ, Kozodaev D, Alexeev A, Escuti MJ, van Heesch C, et al. Photoembossing of periodic relief structures using polymerization- induced diffusion: A combinatorial study. Adv Mater. 2005; 17: 2567-2571.

48. Yang X, Gu M, Wei Q, Zhao W, Zhou G. Preparation of patterned and corrugated alignment layer for liquid crystals and application in light regulating devices. Polymers. In preparation.

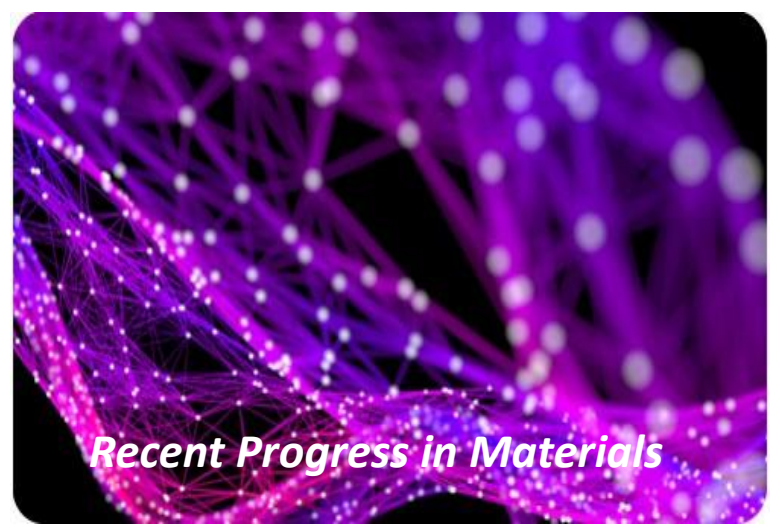

Enjoy Recent Progress in Materials by:

1. Submitting a manuscript

2. Joining in volunteer reviewer bank

3. Joining Editorial Board

4. Guest editing a special issue

For more details, please visit: http://www.lidsen.com/journals/rpm 